\title{
New Approach for Fast Color Image Encryption Using Chaotic Map
}

\author{
Kamlesh Gupta $^{1^{*}}$, Sanjay Silakari ${ }^{2}$ \\ ${ }^{1}$ Department of Computer Science, Jaypee University of Engineering and Technology, Guna, India \\ ${ }^{2}$ Department of Computer Science, Rajiv Gandhi Technical University, Bhopal, India \\ E-mail: $\left\{{ }^{*}\right.$ Kamlesh_rjitbsf, ssilakari\}@yahoo.com \\ Received June 6, 2011; revised July 5, 2011; accepted July 16, 2011
}

\begin{abstract}
Image encryption using chaotic maps has been established a great way. The study shows that a number of functional architecture has already been proposed that utilize the process of diffusion and confusion. However, permutation and diffusion are considered as two separate stages, both requiring image-scanning to obtain pixel values. If these two stages are mutual, the duplicate scanning effort can be minimized and the encryption can be accelerated. This paper presents a technique which replaces the traditional preprocessing complex system and utilizes the basic operations like confusion, diffusion which provide same or better encryption using cascading of 3D standard and 3D cat map. We generate diffusion template using 3D standard map and rotate image by using vertically and horizontally red and green plane of the input image. We then shuffle the red, green, and blue plane by using 3D Cat map and standard map. Finally the Image is encrypted by performing XOR operation on the shuffled image and diffusion template. Theoretical analyses and computer simulations on the basis of Key space Analysis, statistical analysis, histogram analysis, Information entropy analysis, Correlation Analysis and Differential Analysis confirm that the new algorithm that minimizes the possibility of brute force attack for decryption and very fast for practical image encryption.
\end{abstract}

Keywords: Chaotic Map, 3D Cat Map, Standard Map, Confusion and Diffusion

\section{Introduction}

With the fast development of image transmission through computer networks especially the Internet, medical imaging and military message communication, the security of digital images has become a most important concern. Image encryption, is urgently needed but it is a challenging task because it is quite different from text encryption due to some intrinsic properties of images such as huge data capacity and high redundancy, which are generally difficult to handle by using conventional techniques. Nevertheless, many new image encryption schemes have been suggested in current years, among which the chaos-based approach appears to be a hopeful direction.

General permutation-diffusion architecture for chaosbased image encryption was employed in [1,2] as illustrated in Figure 1. In the permutation stage, the image pixels are relocated but their values stay unchanged. In the diffusion stage, the pixel values are modified so that a minute change in one-pixel spreads out to as many pix- els as possible. Permutation and diffusion are two different and iterative stages, and they both require scanning the image in order to gain the pixel values. Thus, in the encryption process, each round of the permutationdiffusion operation requires at least twice scanning the same image.

In this paper, we generate diffusion template using 3D standard map and rotated image by using vertically and horizontally red and green plane of the input image. We then shuffle the red, green, and blue plane by using $3 \mathrm{D}$

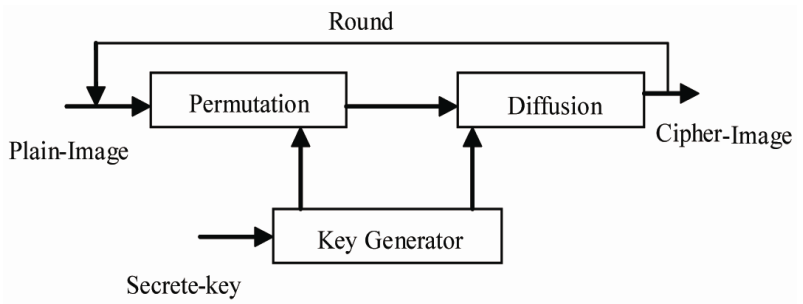

Figure 1. Permutation and diffusion based image cryptosystem. 
Cat map and standard map. Finally the Image is encrypted by performing XOR operation on the shuffled image and diffusion template. The objectives of this new design includes: 1) to efficiently extract good pseudorandom sequences from a cascading of $3 \mathrm{D}$ cat and standard map for color image and 2) to simultaneously perform permutation and diffusion operations for fast encryption.

The rest of this paper is organized as follows: Section 2 focuses on the efficient generation of pseudorandom sequences. In Section 3, proposed algorithm is described in detail. Section 4 presents simulation results and performance analyses. In Section 5, conclusions and future work.

\section{Efficient Generation of Pseudorandom Sequences}

The generation of pseudorandom is based on two cascaded chaotic maps behave as a single chaotic map in present case. The 3D cat map \& 3D standard map are taken for encryption. The pseudorandom matrix generated by this method is given below. (The explanation for pseudorandom sequences generation is given in Section 3).

\section{Proposed Algorithm}

The proposed algorithm are divided into several stages

Table 1. Generation of pseudorandom values by proposed method.

\begin{tabular}{|c|c|c|c|c|c|c|c|c|c|c|c|}
\hline $\mathrm{FC}^{\prime}$ & $E^{\prime}$ & 'C4' & $1^{\prime}$ & 'E6' & 'D3' & 'E8' & 'E9' & 'EA' & 'CB' & 'EA' & $\mathrm{ED}^{\prime}$ \\
\hline $77^{\prime}$ & $C^{\prime}$ & $99^{\prime}$ & $E^{\prime}$ & '5' & $\mathrm{F}^{\prime}$ & $6^{\prime}$ & '17' & '9' & 'EF' & 'FF' & '1B' \\
\hline & & 'A' & 'E2' & 'FA' & '2' & $\mathrm{FC}^{\prime}$ & 'F5' & $' 1 C^{\prime}$ & $' 2 F^{\prime}$ & '1E' & '1F' \\
\hline 2D' & & & '26' & '0' & '28' & '11' & '2A' & '4' & '17' & '15' & 'F8' \\
\hline $9^{\prime}$ & & '15' & '2B' & '3F' & '1E' & '19' & '41' & $2 \mathrm{~A}^{\prime}$ & '1C' & '1D' & '45' \\
\hline $3 C^{\prime}$ & '40' & '10' & '33' & '47' & '44' & '58' & '37' & '5A' & '35' & '5C' & ' $3 F^{\prime}$ \\
\hline $5 \mathrm{~F}^{\prime}$ & '41' & '51' & '5E' & '31' & '54' & '24' & '61' & '35' & '27' & '28' & ' 47 ' \\
\hline $4 \mathrm{D}^{\prime}$ & ' $6 C^{\prime}$ & '4F' & '6E' & '45' & '42' & '56' & '71' & ' $3 \mathrm{~A}^{\prime}$ & '59' & '3C' & '5B' \\
\hline '63' & & '74' & '6D' & '49' & '6F' & '69' & '61' & '6B' & '5D' & '6D' & '6E' \\
\hline $8 \mathrm{~F}^{\prime}$ & '62' & '7B' & '64' & '89' & '66' & '7F' & '8C' & '53' & '54' & '64' & '9F' \\
\hline '80' & '72' & 'A6' & '74' & '99' & '67' & '7D' & '7E' & '9D' & 'AD' & '80' & '81' \\
\hline '87' & & '89' & '99' & '9A' & '9B' & '9C' & 'BB' & $' 70^{\prime}$ & 'AE' & '72' & '73' \\
\hline 'A2' & 'A3' & 'BF' & 'A5' & 'B5' & & 'C2' & '9A' & 'A6' & 'C5' & 'A8' & '9E' \\
\hline '9D' & 'BC' & 'B2' & '90' & 'AC' & 'Al' & 'AE' & 'AF' & 'A0' & 'C8' & 'C9' & 'A3' \\
\hline 'CE' & 'CF' & 'DF' & 'C1' & 'BC' & 'C3' & 'E2' & 'CE' & 'CF' & 'E5' & 'E6' & 'D1' \\
\hline 'E7' & 'F7' & 'CE' & 'DB' & 'D8' & 'DD' & 'DA' & 'BD' & 'DC' & 'C5' & 'E4' & 'B1' \\
\hline
\end{tabular}

and explained below.

\subsection{Diffusion Template}

According to the proposal the diffusion template must have the same size as main image. Let the main image have $m$ number of rows $n$ number of columns then the diffusion template is created as follows

$$
(i, j, k)=\operatorname{round}\left(\frac{255}{n} \times j\right)
$$

where $1 \leq i \leq m, 1 \leq j \leq n$ and $1 \leq k \leq 3$.

Equation (1) form the matrix with all rows filled with linearly spaced number in between 0 to 255 . The sequence is randomized by $3 \mathrm{D}$ standard map in discrete form as given below.

The 3D standard map randomizes the pixels by reallocating it in new position by utilizing its property of one to one mapping. Figure 2 shows the final diffusion template by using 3D standard map.

$$
\begin{gathered}
i^{\prime}=(i+j) \bmod m \\
j^{\prime}=\left[j+k+K 1 \times \sin \left(i \times \frac{c}{2 \times p i}\right)\right] \bmod n \\
k^{\prime}\left[k+K 1 \times \sin \left(i \times \frac{p}{2 \times p i}\right)+K 2 \times \sin \left(j \times \frac{p}{2 \times p i}\right)\right] \bmod p
\end{gathered}
$$

where the $K 1, K 2$ are the integers, $p=3$ for the case of color image and $i^{\prime}, j^{\prime}, k^{\prime}$ shoes the transformed location of $i, j, k$.

$$
I_{\text {diff }}^{\prime}\left(i^{\prime}, j^{\prime}, k^{\prime}\right)=I_{\text {diff }}^{\prime}(i, j, k)
$$

\subsection{Image Encryption}

Step 1. The main image is divided into three separate images $I_{R}, I_{G}$ and $I_{B}$ as follows

$$
\begin{gathered}
I_{R}(x, y)=I(x, y, 1) \quad I_{G}(x, y)=I(x, y, 2) \\
I_{B}(x, y)=I(x, y, 3)
\end{gathered}
$$

where $\quad 1 \leq x \leq m$ and $1 \leq y \leq n$.

Step 2. The Red and Green image are transform verti-

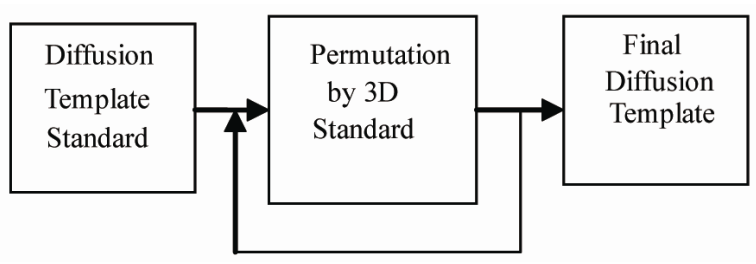

Figure 2. Diffusion template. 
cally and horizontally respectively. The blue image remains same and reconstructs the new image.

$$
\begin{gathered}
I_{R}^{\prime}(x, y)=I_{R}\left[\left(x+\frac{m}{2}\right)\right] \bmod m, y \\
I_{G}^{\prime}(x, y)=I_{R}\left[x\left(y+\frac{n}{2}\right)\right] \bmod n \\
I_{B}^{\prime}(x, y)=I_{B}(x, y) \quad I_{\text {new }}(x, y, 1)=I_{R}^{\prime}(x, y) \\
I_{\text {new }}(x, y, 2)=I_{G}^{\prime}(x, y) \quad I_{\text {new }}(x, y, 3)=I_{B}^{\prime}(x, y) .
\end{gathered}
$$

Step 3. Perform the first level confusion by using 2D cat map. Slice the plane normal to $R, G, B$ Planes by

$$
I_{\text {new }}^{\prime}(i, j, k)=I_{\mathrm{SRGB}}^{\prime}\left(j^{\prime}, k^{\prime}\right)=I_{\text {new }}(i, j, k)
$$

where $1 \leq i \leq m, 1 \leq j \leq n$ and $1 \leq k \leq 3$.

$$
\begin{gathered}
j^{\prime}=\left(j+r_{x}+r_{y}+p \times k\right) \bmod m \\
k^{\prime}=\left(q \times j+r_{y}+(p \times q+1) \times k\right) \bmod n
\end{gathered}
$$

where $j^{\prime}$ and $k^{\prime}$ are obtained by 2D cat map and $p$ and $q$ are integer $>0$ and $r_{x}, r_{y}$ are offset integer such that $0 \leq r_{x} \leq m$, $0 \leq r_{y} \leq n$.

Step 4. Generate Final confusion stage by two cascade 3D maps first by cat map then by standard map. So the transformation of location $(i, j, k)$ into $\left(i^{\prime \prime}, j^{\prime \prime}, k^{\prime \prime}\right)$ is performed by following equations.

$$
\begin{gathered}
i^{\prime}=\left[\left(1+a_{x} a_{z} b_{y}\right) \times i+a_{z} \times j\right. \\
\left.+\left(a_{y}+a_{x} \times a_{z}+a_{x} \times a_{y} \times a_{z} \times b_{y}\right) \times k\right] \bmod m \\
i^{\prime}=\left[\left(b_{z}+a_{x} \times b_{y}+a_{x} \times a_{z} \times b_{y} \times b_{z}\right) \times i+\left(a_{z} \times b_{z}+1\right)\right. \\
\times j+\left(a_{y} \times a_{z}+a_{x} \times a_{y} \times a_{z} \times b_{y} \times b_{z}+a_{x} \times a_{z} \times b_{y}\right. \\
\left.\left.+a_{y} \times b_{y}+1\right) \times k\right] \bmod n \\
k^{\prime}=\left[\left(a_{x} \times b_{x} \times b_{y}+b_{y}\right) \times i+b_{x} \times j\right. \\
\left.+\left(a_{x} \times a_{y} \times b_{x} \times b_{y}+a_{x} \times b_{x}+a_{y} \times b_{y}+1\right) \times k\right] \bmod p \\
i^{\prime \prime}=\left[\left(i^{\prime}+k^{\prime}\right)\right] \bmod m \\
j^{\prime \prime}=\left[\left(i^{\prime}+j^{\prime}\right)+K 1 \times \sin \left(i^{\prime \prime} \times \frac{n}{2 \times p i}\right)\right] \bmod n \\
\quad\left[\begin{array}{c}
k^{\prime \prime}=\left[k^{\prime}+K 1 \times \sin \left(i^{\prime \prime} \times \frac{p}{2 \times p i}\right)\right. \\
+
\end{array}\right) \\
\left.\quad K 2 \times \sin \left(j+\frac{p}{2 \times p i}\right)\right] \bmod p
\end{gathered}
$$

where $a_{x}, a_{y}, a_{z}, b_{x}, b_{y}, b_{z}$ and $K 1, K 2$ are integers $>0$.

Each confusion step is followed by diffusion obtained by EXOR operations performed between each pixels of $I_{\text {conf }}$ and diffusion $I_{\text {diff. }}$. The proposed image encryption architecture is given in Figure 3.

$$
I_{\text {encp }}=I_{\text {conf }} \oplus I_{\text {diff }} \text {. }
$$

\subsection{Key Generation Process}

The proposed method has a large number of variables which can be used as key parameters but to avoid the exceptionally large key and decreased key sensitivity, the parameter which does not having great affects on encryption are avoid or scaled. The selected key parameters and their length are given below

Step 1. Diffusion template shuffling $\mathbf{D}_{\mathbf{s}}=8$ bits.

Step 2. Diffusion template offset value $\mathbf{D}_{\mathbf{x}} \mathbf{D}_{\mathbf{y}} \mathbf{D}_{\mathbf{z}}=8+$ $8+2=18$ bits.

Step 3. Diffusion template variables $\mathbf{D}_{\mathbf{k} 1} \mathbf{D}_{\mathbf{k} 2}=8+8=$ 16 bits.

Step 4. Sliced RGB plane Shuffling $=\mathbf{S}_{\mathbf{s}}=8$ bits.

Step 5. Sliced RGB plane offset values $\mathbf{S}_{\mathbf{x}} \mathbf{S}_{\mathbf{y}}=8$ bits.

Step 6. Sliced RGB Plane Variables $\mathbf{S}_{\mathbf{p}} \mathbf{S}_{\mathbf{q}}=8+8=16$ bits.

Step 7. Final Confusion shuffling $\mathbf{C}_{\mathbf{s}}=8$ bits.

Step 8. Confusion offset of cat map $\mathbf{C}_{\mathbf{x}} \mathbf{C}_{\mathbf{y}} \mathbf{C}_{\mathbf{z}}=8+8+$ $2=18$ bits.

Step 9. Confusion cat map variables $\mathbf{C}_{\mathbf{a}} \mathbf{C}_{\mathbf{b}}=8+8=$ 16 bits.

Step 10. Confusion offset of standard map $\mathbf{C}_{\mathbf{x}}{ }^{\prime} \mathbf{C}_{\mathbf{y}}{ }^{\prime} \mathbf{C}_{\mathbf{z}}{ }^{\prime}$ $=8+8+2=18$ bits.

Step 11. Confusion standard map variables $\mathbf{C}_{\mathbf{k} 1} \mathbf{C}_{\mathbf{k} 2}=8$ $+8=16$ bits.

\section{Final key structure}

$$
\begin{aligned}
& D_{\mathrm{s}} D_{\mathrm{x}} D_{\mathrm{y}} D_{\mathrm{z}} D_{\mathrm{k} 1} \mathrm{D}_{\mathrm{k} 2} \mathrm{~S}_{\mathrm{s}} S_{\mathrm{x}} S_{\mathrm{y}} S_{\mathrm{p}} S_{\mathrm{q}} \\
& \mathrm{C}_{\mathrm{s}} \mathrm{C}_{\mathrm{x}} \mathrm{C}_{\mathrm{y}} \mathrm{C}_{\mathrm{z}} \mathrm{C}_{\mathrm{a}} \mathrm{C}_{\mathrm{b}} \mathrm{C}_{\mathrm{x}}{ }^{\prime} \mathrm{C}_{\mathrm{y}}{ }^{\prime} \mathrm{C}_{\mathrm{z}}{ }^{\prime} \mathrm{C}_{\mathrm{k} 1} \mathrm{C}_{\mathrm{k} 2} \\
& \text { Total bits }=8+18+16+8+8+16+8 \\
& +16+16+18+16=148 \text { bits. }
\end{aligned}
$$

\subsection{Image Decryption}

Step 1. Generate the diffusion template in same way as in encryption section.

Step 2. Re-transformation of location is done by two cascaded 3D maps firstly by standard map then by cat map.

So the re-transformation of location $\left(i^{\prime \prime}, j^{\prime \prime}, k^{\prime \prime}\right)$ into $(i, j$, $k$ ) is performed by following equations

$$
\begin{gathered}
i^{\prime}=\left[\left(i^{\prime \prime}+k^{\prime \prime}\right)\right] \bmod m \\
j^{\prime}=\left[i^{\prime \prime}+j^{\prime \prime}+k 1 \times \sin \left(i^{\prime} \times \frac{n}{2 \times p i}\right)\right] \bmod n
\end{gathered}
$$




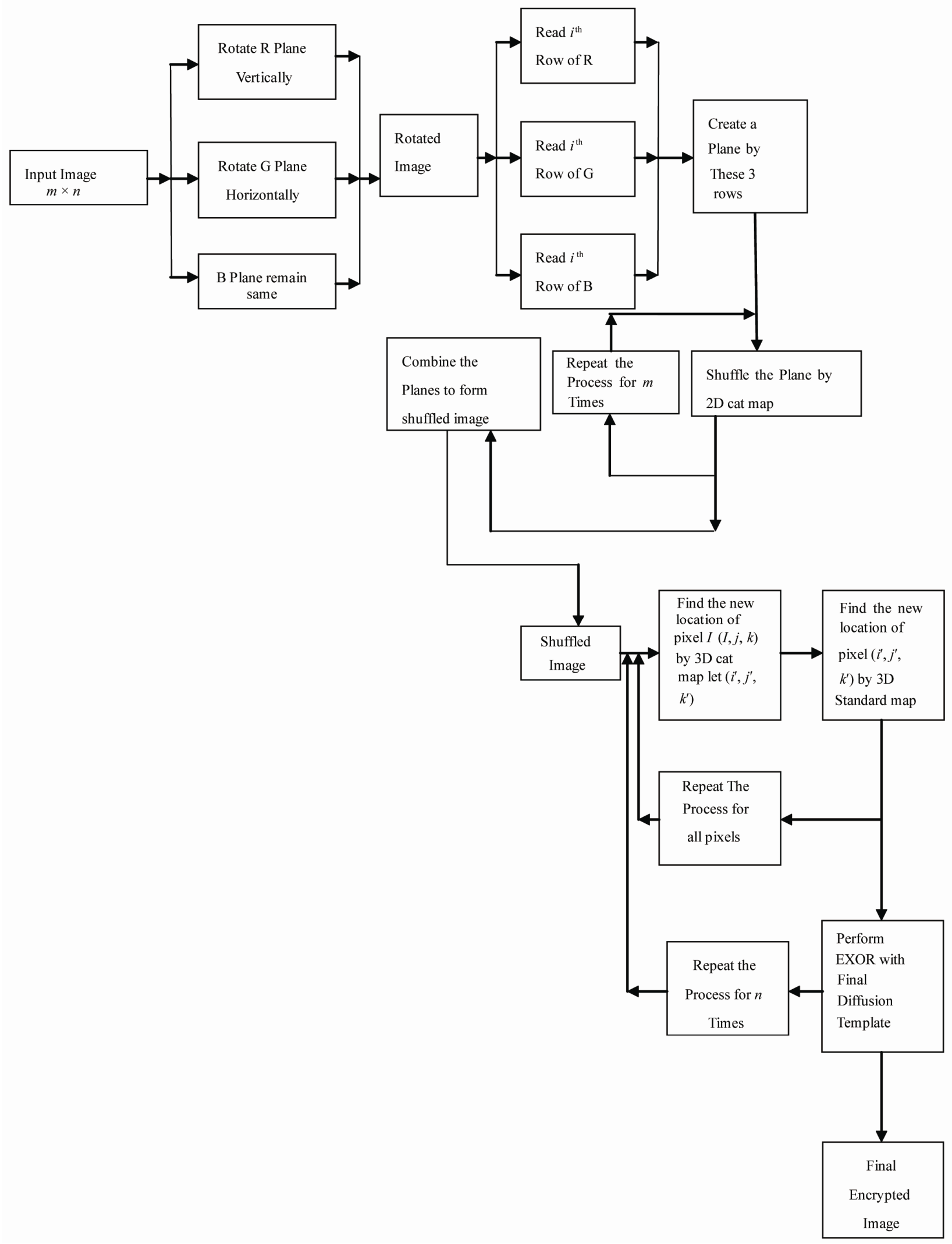

Figuer 3. Image encryption by using confusion and diffusion. 


$$
\begin{aligned}
& k^{\prime}= {\left[k^{\prime \prime}+k 1 \times \sin \left(j^{\prime} \times \frac{n}{2 \times p i}\right)+k 2 \times\left(j^{\prime} \times \frac{n}{2 \times p i}\right)\right] \bmod p } \\
& i= {\left[\left(1+a_{x} a_{z} b_{y}\right) \times i^{\prime}+a_{z} \times j^{\prime}+\right.} \\
&\left.\left(a_{y}+a_{x} \times a_{z}+a_{x} \times a_{y} \times a_{z} \times b_{y}\right) \times k^{\prime}\right] \bmod m \\
& i=\left(b_{z}+a_{x} \times b_{y}+a_{x} \times a_{z} \times b_{y} \times b_{z}\right) \times i^{\prime}+\left(a_{z} \times b_{z}+1\right) \\
& \times j^{\prime}+\left(a_{y} \times a_{z}+a_{x} \times a_{y} \times a_{z} \times b_{y} \times b_{z}+a_{x} \times a_{y} \times b_{y}\right. \\
&\left.\left.+a_{x}\right) \times k^{\prime}\right] \bmod n \\
& k=\left(a_{x} \times b_{x} \times b_{y}+b_{y}\right) \times i^{\prime}+b_{x} \times j^{\prime}+ \\
&\left.\left(a_{x} \times a_{y} \times b_{x} \times b_{y}+a_{x} \times b_{x}+a_{y} \times b_{y}+1\right) \times k^{\prime}\right] \bmod p \\
& I_{\text {retransf }}(i, j, k)=I_{\text {encp }}(i, j, k)
\end{aligned}
$$

where $a_{x}, a_{y}, a_{z}, b_{x}, b_{y}, b_{z}$ and $K 1, K 2$ are integers $>0$.

Each confusion step is followed by diffusion obtained by EXOR operations performed between each pixels of $I_{\text {retrnsf }}$ and diffusion $I_{\text {diff }}$

$$
I_{\text {dencp }}^{\prime}=I_{\text {retrnsf }} \oplus I_{\text {diff }} .
$$

Step 3. Performing inverse of First level confusion.

Slicing the plane normal to $R, G, B$ Planes

$$
I_{\mathrm{SRGB}}^{\prime}(j, k)=I_{\text {dencp }}^{\prime}(i, j, k)
$$

for each value of $i, j$ changed from 0 to $m, k$ changed from 0 to 3 .

De-shuffling the sliced plane

$$
I_{\mathrm{DRGB}}(j, k)=I_{\mathrm{SRGB}}^{\prime}(j, k)
$$

where $j^{\prime}$ and $k^{\prime}$ are obtained by 2D cat map given below

$$
\begin{gathered}
j^{\prime}=\left(j+r_{x}+r_{y}+p \times k\right) \bmod m \\
k^{\prime}=\left(q \times j+r_{y}+(p \times q+1) \times k\right) \bmod n
\end{gathered}
$$

where $p$ and $q$ are integers $>0$, and $r_{x}, r_{y}$ are offset integers such that $0 \leq r_{x} \leq \mathrm{m}$ and $0 \leq r_{y} \leq n$.

Recombining the planes for forming $3 \mathrm{D}$ matrix for next operation

$$
I^{\prime}(i, j, k)=I_{\mathrm{DRGB}}^{\prime}(j, k) .
$$

Step 4. Re-rotating the image planes

Dividing main image into three separate images $I_{R}, I_{G}$ and $I_{B}$ as follows

$$
\begin{gathered}
I_{R}(x, y)=I^{\prime}(x, y, 1) \quad I_{g}(x, y)=I^{\prime}(x, y, 2) \\
I_{B}(x, y)=I^{\prime}(x, y, 3)
\end{gathered}
$$

where $1 \leq x \leq m$ and $1 \leq y \leq n$.

Scrolling the red plane vertically

$$
I_{R}(x, y)=I_{R}\left[\left(x+\frac{m}{2}\right) \bmod m, y\right] .
$$

Scrolling the green plane horizontally

$$
I_{G}(x, y)=I_{R}\left[x\left(y+\frac{n}{2}\right) \bmod n\right] .
$$

Blue plane remain intact.

$$
I_{B}(x, y)=I_{B}(x, y) .
$$

Step 5. Next recombination of planes are performed to form final decrypted image

$$
\begin{gathered}
I_{\text {final }}(x, y, 1)=I_{R}(x, y) \quad I_{\text {final }}(x, y, 2)=I_{G}(x, y) \\
I_{\text {final }}(x, y, 3)=I_{B}(x, y)
\end{gathered}
$$

\section{Performance Analysis}

\subsection{Key Space Analysis}

The strong point of the proposed algorithm is the generation of the permutation sequence with the chaos sequence. The key space should also be suitably large to make brute-force attack not feasible. In the proposed algorithm, we use 148 bit key (37 Hex number) is used. It has been observed in Figures 4(a) and (b) that with slightly varying the initial condition of the chaotic sequence. It has been almost impossible to decrypt the image.

\subsection{Statistical Analysis}

It is well known that passing the statistical analysis on cipher-text is of crucial importance for a cryptosystem actually, an ideal cipher should be strong against any statistical attack. In order to prove the security of the proposed image encryption scheme, the following Statistical tests are performed.

\subsubsection{Histogram Analysis}

To prevent the access of information to attackers, it is important to ensure that encrypted and original images do not have any statistical similarities. The histogram analysis clarifies that, how the pixel values of image are distributed. A number of images are encrypted by the encryption schemes under study and visual test is performed.

An example is shown in Figure 5. In Figure 5 shows histogram analysis on test image using proposed algorithm. The histogram of original image contains great sharp rises followed by sharp declines as shown in Figure 5 and the histograms of the encrypted images for different round as shown in Figures 5(a)-(f) have uniform distribution which is significantly different from original image and has no statistical similarity in ap- 


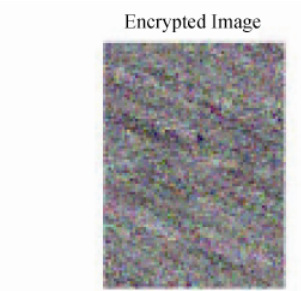

03010020304020301011010110D2833020202

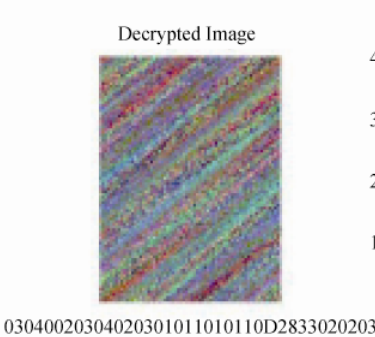

03040020304020301011010110D2833020203
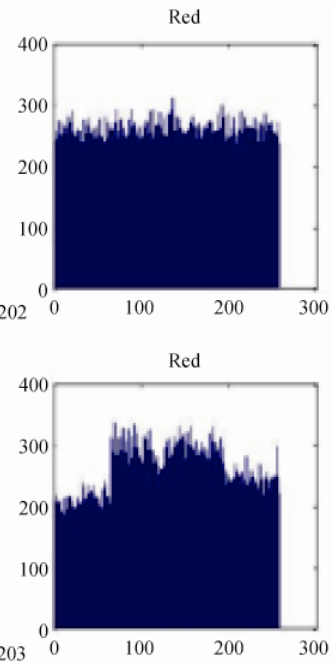

(a)
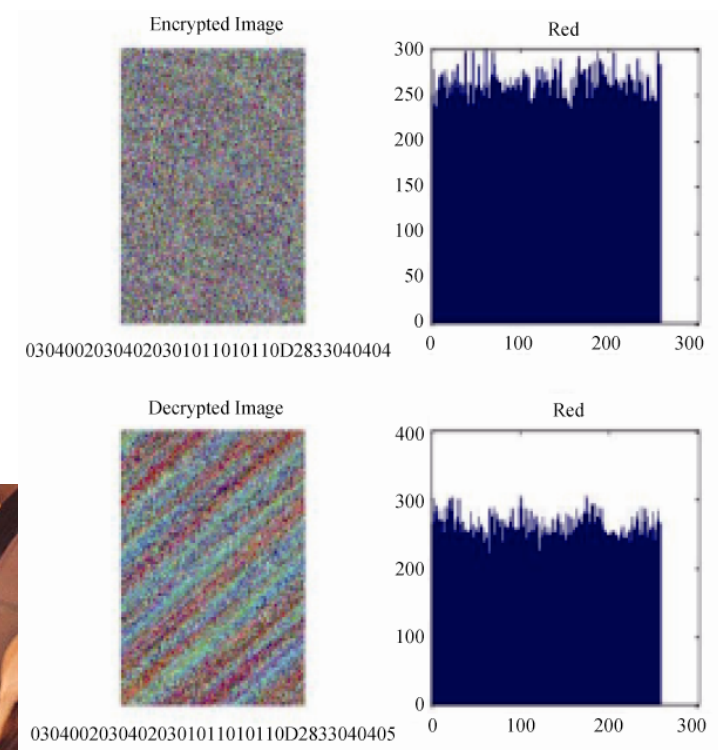
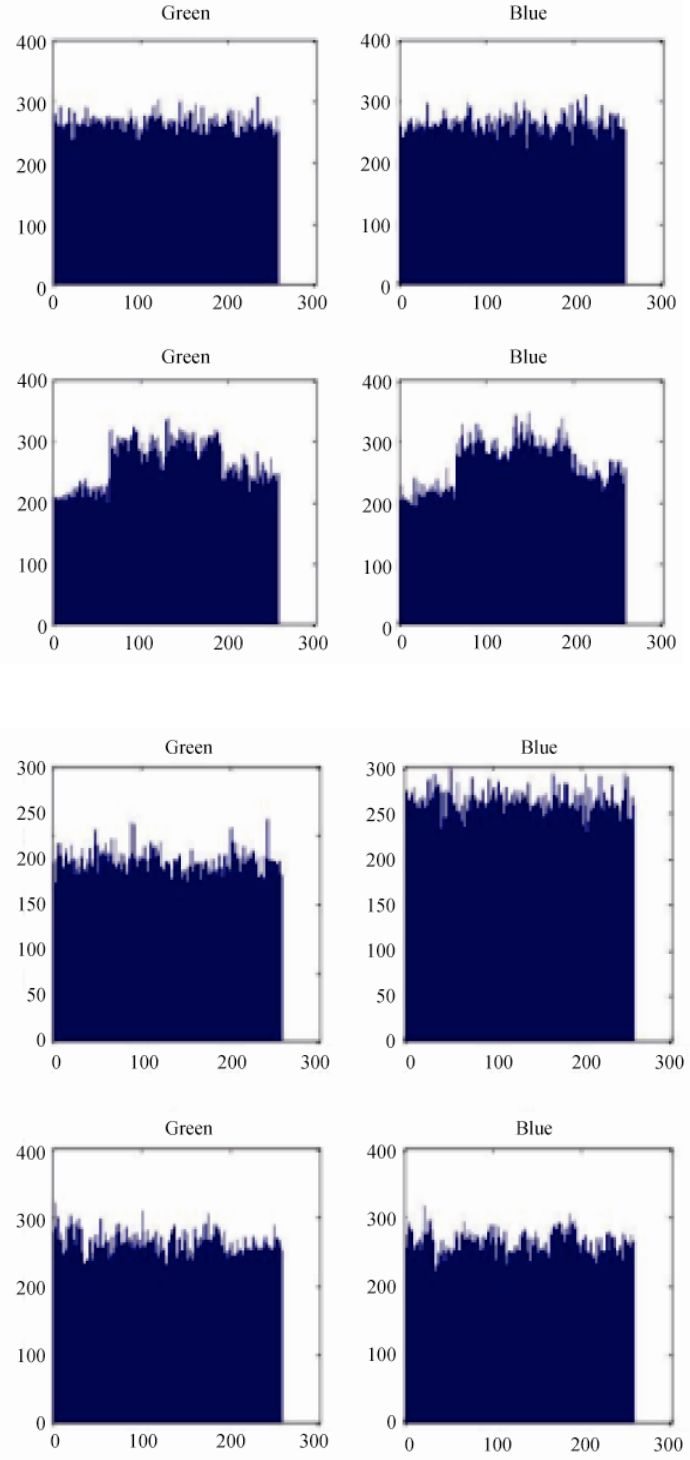

(b)

Figure 4. (a) Input image encrypted with $03040020304020301011010110 D 2833020202$ and Decrypted by 0304002030402030 1011010110D2833020203; (b) Input lenna image encrypted with 03040020304020301011010110D2833040404 and Decrypted by $03040020304020301011010110 \mathrm{D} 2833040405$.

pearance. So, the surveyed algorithms do not provide any clue for statistical attack. The encrypted image histogram, approximated by a uniform distribution, is quite different from plain-image histogram.

\subsubsection{Correlation Analysis}

There is a very good correlation among adjacent pixels in the digital image [3]. Equation (5) is used to study the
Correlation between two adjacent pixels in horizontal, vertical and diagonal orientations. This is shown in Figure 6.

$x$ and $y$ are intensity values of two neighboring pixels in the image and $N$ is the number of adjacent pixels selected from the image to calculate the correlation. 1000 pairs of two adjacent pixels are selected randomly from image to test correlation. The correlation coefficient be-

$$
c_{r}=\frac{N \sum_{j=1}^{N}\left(x_{j} \times y_{j}\right)-\sum_{j=1}^{N} x_{j} \times \sum_{j=1}^{N} y_{j}}{\sqrt{\left(N \sum_{j=1}^{N} x_{j}^{2}-\left(\sum_{j=1}^{N} x_{j}\right)^{2}\right) \times\left(N \sum_{j=1}^{N} y_{j}^{2}-\left(\sum_{j=1}^{N} y_{j}\right)^{2}\right)}}
$$




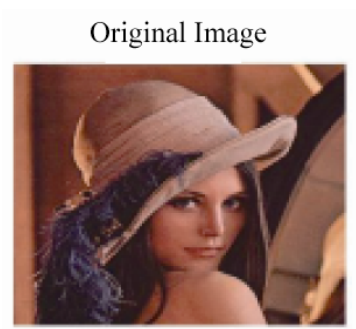

Encrypted Image
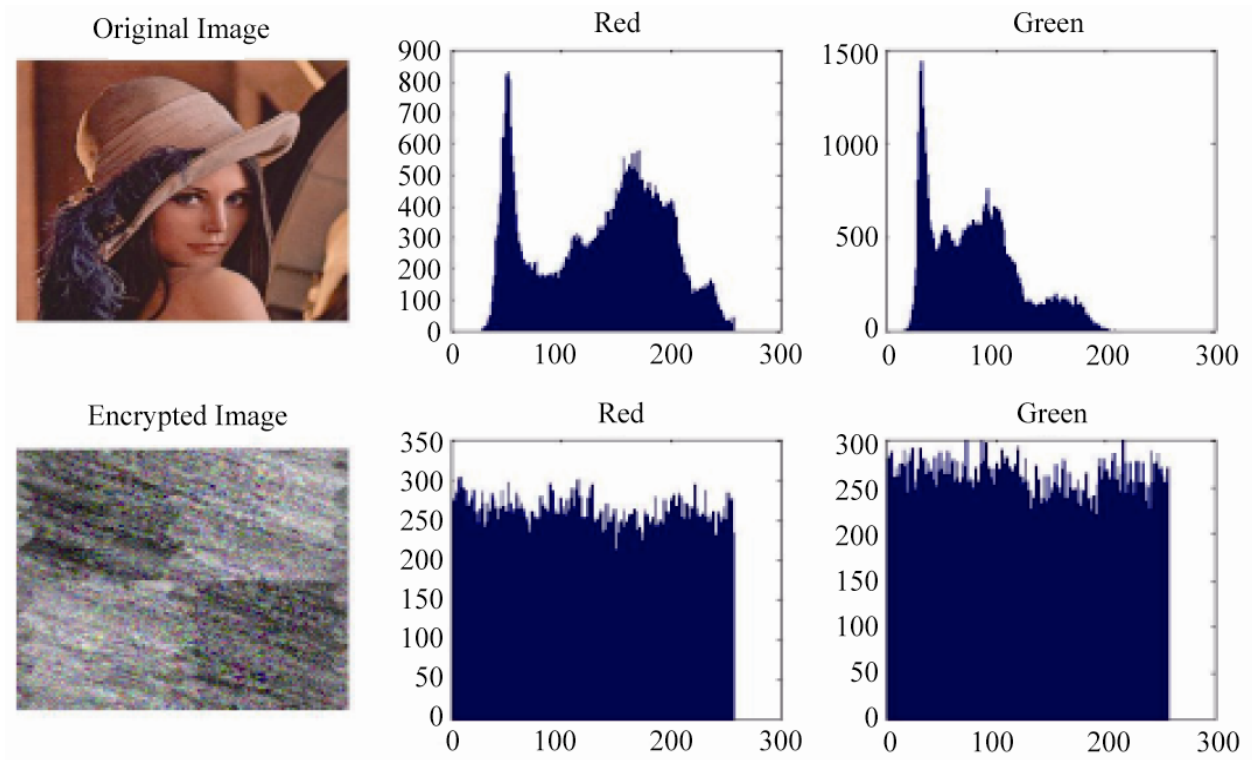

(a)
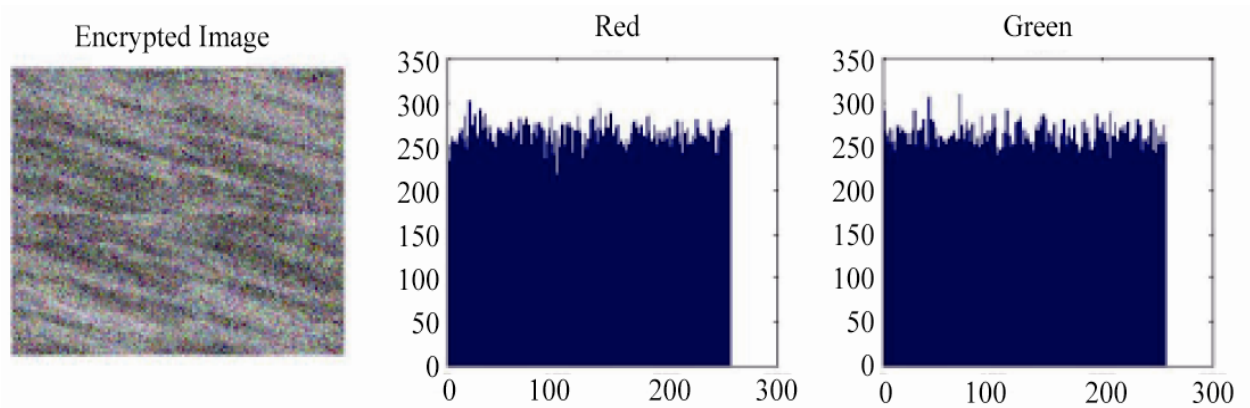

(b)
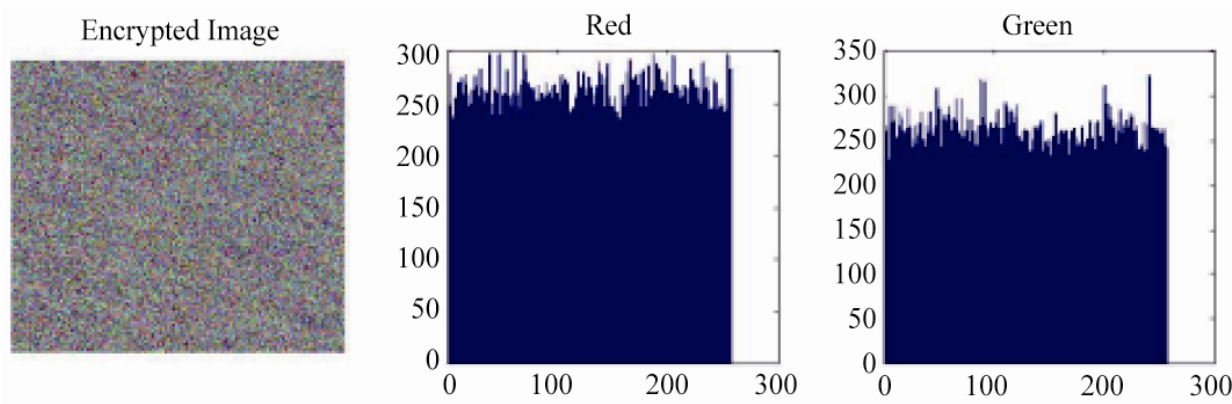

(c)

Encrypted Image
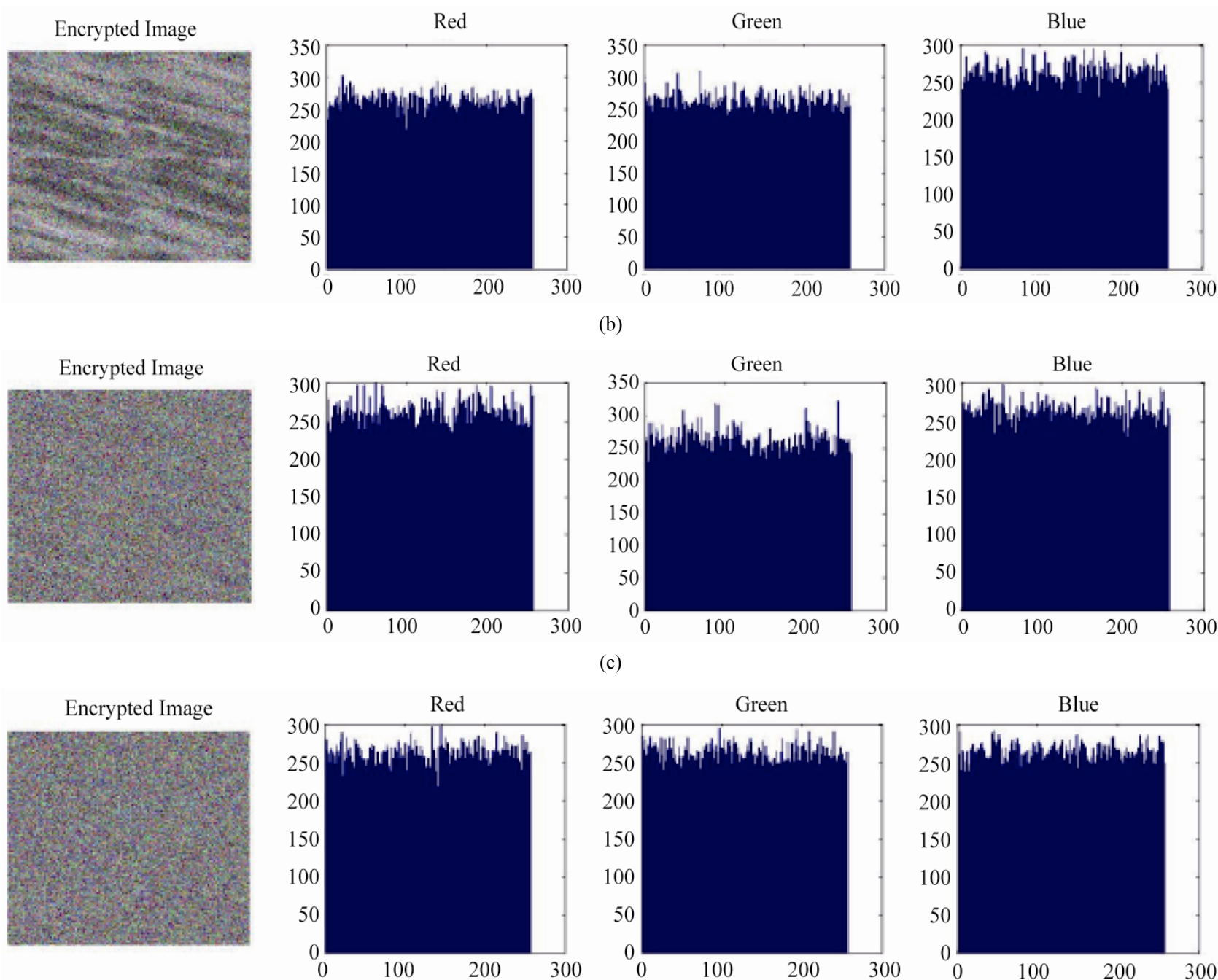

(d)
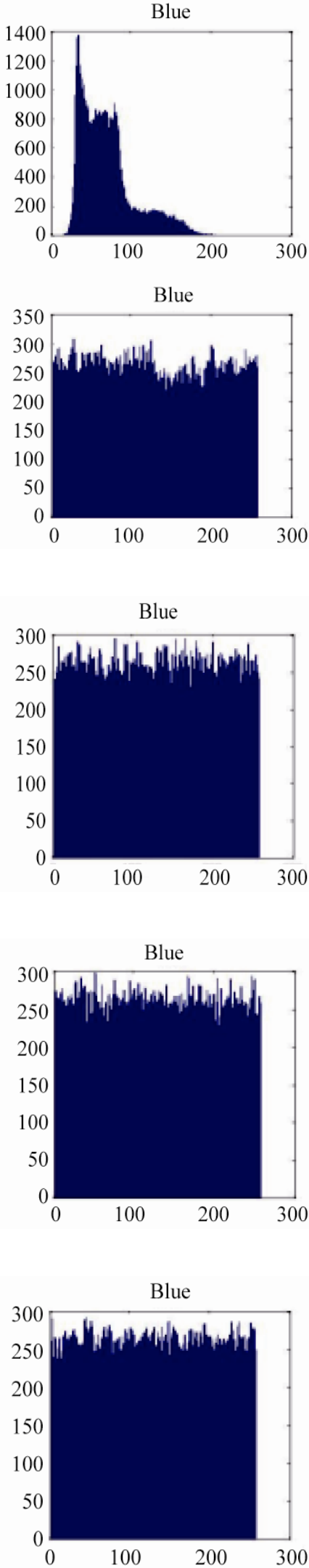

Copyright (C) 2011 SciRes. 

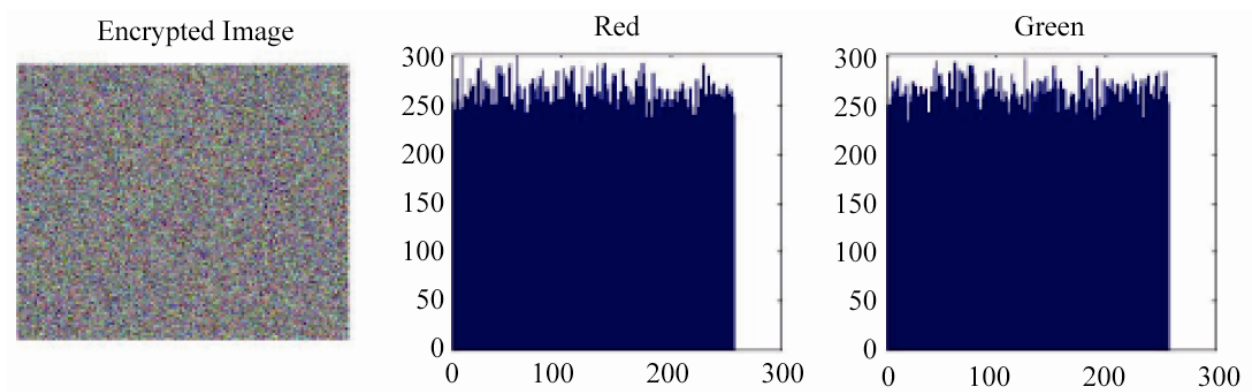

(e)
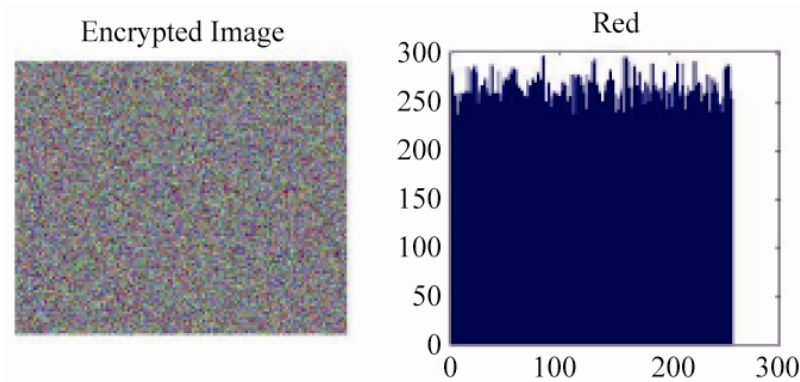
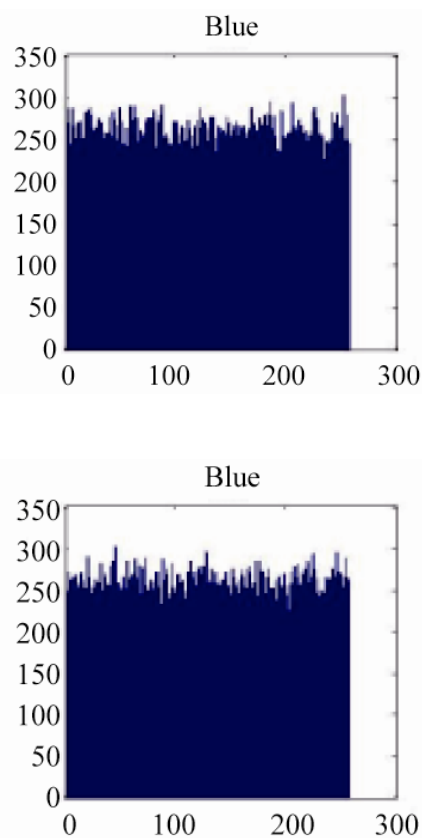

(f)

Figure 5. (a) Histogram for red, green and blue plane of original and encrypted image for $R=1$; (b) Histogram for red, green and blue plane of encrypted image for $R=2$; (c) Histogram for red, green and blue plane of encrypted image for $R=4$; (d) Histogram for red, green and blue plane of encrypted image for $R=8$; (e) Histogram for red, green and blue plane of encrypted image for $R=16$; (f) Histogram for red, green and blue plane of encrypted image for $R=32$.

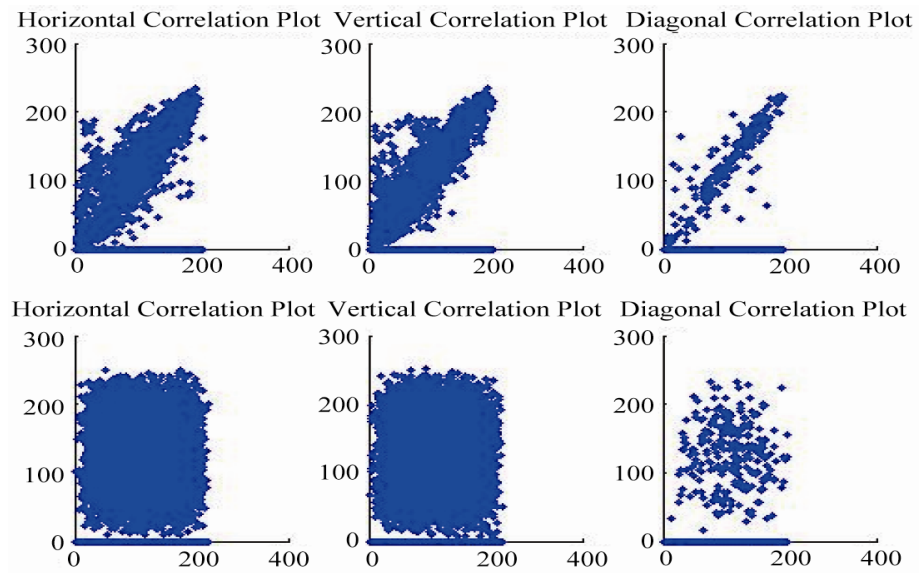

Figure 6. Correlation for horizontal, vertical and diagonal.

tween original and cipher image is calculate in Table 6.

\subsection{Key Space Analysis}

Key space size is the total number of different keys that can be used in the cryptography. Cryptosystem is totally sensitive to all secret keys. A good encryption algorithm should not only be sensitive to the cipher key, but also the key space should be large enough to make brute-force attack infeasible. The key space size for initial conditions and control parameters is over than $2^{148}$. Apparently, the key space is sufficient for reliable practical use.

\subsection{Differential Analysis}

In general, a desirable characteristic for an encrypted image is being sensitive to the little changes in plainimage (e.g. modifying only one pixel). Adversary can create a small change in the input image to observe changes in the result. By this method, the meaningful relationship between original image and cipher image can be found. If one little change in the plain-image can 
cause a significant change in the cipher-image, with respect to diffusion and confusion, then the differential attack actually loses its efficiency and becomes almost useless. There are three common measures were used for differential analysis: MAE, NPCR and UACI. Mean Absolute Error (MAE). The bigger the MAE value, the better the encryption security. NPCR means the Number of Pixels Change Rate of encrypted image while one pixel of plain-image is changed. UACI which is the Unified Average Changing Intensity, measures the average intensity of the differences between the plain-image and Encrypted image.

Let $C(i, j)$ and $P(i, j)$ be the color level of the pixels at the $i$ th row and $j$ th column of a $W \times H$ cipher and plainimage, respectively. The MAE between these two images is defined in

$$
\mathrm{MAE}=\frac{1}{W \times H} \sum_{j=1}^{W} \sum_{i=1}^{H}|c(i, j)-p(i, j)| .
$$

Consider two cipher-images, $C 1$ and $C 2$, whose corresponding plain-images have only one pixel difference.

The NPCR of these two images is defined in

$$
\mathrm{NPCR}=\frac{\sum_{i, j} D(i, j)}{W \times H} \times 100 \%
$$

where $W$ and $H$ are the width and height of the image and $D(i, j)$ is defined as

$$
D(i, j)=\left\{\begin{array}{ll}
0, & \text { if } C 1(i, j)=C 2(i, j) \\
1, & \text { if } C 1(i, j) \neq C 2(i, j)
\end{array} .\right.
$$

Another measure, UACI, is defined by the following formula:

$$
\mathrm{UACI}=\frac{1}{W \times H} \sum_{i, j}\left[\frac{c 1(i, j)-c 2(i, j)}{255}\right] \times 100 \% .
$$

Tests have been performed on the encryption schemes on a 256-level color image of size $256 \times 256$ shown in Figures 5(a)-(f). The MAE, NPCR and UACI experiment result is shown in Tables 4 and 2. The Tables 3 and $\mathbf{5}$ compare the result of Yong previous related work based on chaotic map and our. Results obtained from NPCR show that the encryption scheme's sensitivity to little changes in the input image is under $0.01 \%$. According to the UACI estimation result, the rate influence due to one pixel change is very low. The results demonstrate that a swiftly change in the original image will result in a negligible change in the ciphered image.

\subsection{Information Entropy Analysis}

It is well known that the entropy $H(m)$ of a message source $\mathrm{m}$ can be measured by

$$
H(m)=\sum_{i=0}^{m-1} p\left(m_{i}\right) \log \frac{1}{p\left(m_{i}\right)}
$$

where $M$ is the total number of symbols $m_{i} \in m ; p\left(m_{\mathrm{i}}\right)$ represents the probability of occurrence of symbol mi and $\log$ denotes the base 2 logarithm so that the entropy is expressed in bits. For a random source emitting 256 symbols, its entropy is $H(m)=8$ bits. This means that the cipher-images are close to a random source and the proposed algorithm is secure against the entropy attack. The test result on different image for different round is defined in Table 7.

\subsection{Speed Analysis}

Apart from the security consideration, some other issues on image encryption are also important. This includes the encryption speed for real-time processes. In general, encryption speed is highly dependent on the CPU/MPU structure, RAM size, Operating System platform, the programming language and also on the compiler options. So, it is senseless to compare the encryption speeds of two ciphers image.

Without using the same developing atmosphere and optimization techniques. Inspire of the mentioned difficulty, in order to show the effectiveness of the proposed image encryption scheme over existing algorithms. We

Table 2. NPCR, UACI and Entropy for key sensitivity test.

\begin{tabular}{cccc}
\hline $\begin{array}{c}\text { Lenna Error } \\
\text { Image }\end{array}$ & $R=2$ & $R=3$ & $R=4$ \\
\hline NPCR & 99.5966593424 & 99.6098836263 & 99.651082356 \\
UACI & 52.5394813687 & 51.6816741344 & 50.603535970 \\
Entropy & 7.99913068980 & 7.99912231127 & 7.9991865161 \\
& \multicolumn{3}{c}{ Baboon Error Image } \\
NPCR & 99.6103922526 & 99.5905558268 & 99.599711100 \\
UACI & 46.9998348460 & 47.8581327550 & 48.719709807 \\
Entropy & 7.99901078968 & 7.99905756543 & 7.9991734549 \\
\hline
\end{tabular}

Table 3. Comparison of NPCR and UACI with Yong Wong et al.

\begin{tabular}{cccccccc}
\hline Name of image & & \multicolumn{2}{c}{$R=1$} & & \multicolumn{2}{c}{$R=3$} \\
\hline \multirow{3}{*}{ Airplane } & & Our & Yong Wang et. al & Our & Yong Wang et. al & Our & Yong Wang et al. \\
& NPCR & $\mathbf{9 9 . 6 2}$ & 97.621 & 99.60 & 99.637 & 99.62 \\
& UACI & $\mathbf{3 3 . 1 9}$ & 32.909 & 33.10 & 33.575 & 33.35 \\
\hline
\end{tabular}


Table 4. NPCR and UACI for different round on different color image.

\begin{tabular}{|c|c|c|c|c|c|c|c|c|c|}
\hline Image & & $R=1$ & $R=2$ & $R=3$ & $R=4$ & $R=8$ & $R=10$ & $R=16$ & $R=32$ \\
\hline \multirow{3}{*}{ Baboon } & NPCR & 99.55 & 99.57 & 99.59 & 99.59 & 99.61 & 99.60 & 99.60 & 99.61 \\
\hline & UACI & 37.17 & 38.68 & 39.00 & 38.78 & 38.69 & 38.88 & 39.03 & 38.89 \\
\hline & MAE & 71.65 & 74.52 & 75.29 & 75.18 & 75.23 & 75.37 & 75.34 & 75.50 \\
\hline \multirow{3}{*}{ Lenna } & NPCR & 99.63 & 99.64 & 99.59 & 99.62 & 99.62 & 99.61 & 99.62 & 99.59 \\
\hline & UACI & 28.87 & 27.51 & 27.33 & 27.42 & 27.43 & 27.64 & 27.51 & 27.37 \\
\hline & MAE & 80.84 & 77.24 & 77.46 & 77.58 & 77.76 & 77.84 & 77.67 & 77.54 \\
\hline \multirow{3}{*}{ Pepper } & NPCR & 99.62 & 99.62 & 99.58 & 99.58 & 99.63 & 99.62 & 99.62 & 99.62 \\
\hline & UACI & 38.05 & 38.26 & 37.99 & 38.03 & 38.34 & 38.20 & 38.33 & 38.11 \\
\hline & MAE & 75.20 & 74.68 & 74.27 & 74.48 & 74.89 & 74.62 & 74.62 & 74.61 \\
\hline \multirow[b]{2}{*}{ Airplane } & NPCR & 99.62 & 99.60 & 99.59 & 99.62 & 99.63 & 99.61 & 99.59 & 99.60 \\
\hline & UACI & 33.19 & 33.10 & 3335 & 3327 & 33,329 & 33.28 & 33,32 & 33,31 \\
\hline
\end{tabular}

Table 5. The round number of scanning-image, permutation and diffusion to achieve NPCR $>0.996$ and UACI > 0.287 .

\begin{tabular}{cccc}
\hline & NPCR & UACI & $\begin{array}{c}\text { No. of Round for Confusion } \\
\text { and Diffusion }\end{array}$ \\
\hline Our & $>0.996$ & $>0.287$ & 1 \\
Ref. [3] & $>0.996$ & $>0.333$ & 2 \\
Ref. [4] & $>0.996$ & $>0.333$ & 18 \\
Ref. [5] & $>0.996$ & & 5 \\
Ref. [6] & $>0.996$ & $>0.333$ & 6 \\
Ref. [7] & $>0.996$ & $>0.333$ & 6 \\
\hline
\end{tabular}

evaluated the performance of encryption schemes with an un-optimized MATLAB 7.0 code. Performance was measured on a machine with Intel core 2 Duo $2.00 \mathrm{GHz}$ CPU with 2 GB of RAM running on Windows XP. The time for encryption and decryption is measured for different round is shown in Tables 8 and 9.

\subsection{FIPS 140 Testing}

We also show that our proposed algorithm pass the FIPS 140-2 randomness tests. There are four tests: Mono-bit, Poker, Runs tests and Long run tests. Each of the tests was designed to test the randomness of a sample sequence length of 20,000 bits as follows:

\subsubsection{The Monobit Test}

1) Calculate $x$ which is the number of ones in the 20,000 bit stream.

2) The test is passed if $9725<x<10,275$.

\subsubsection{The Poker Test}

1) Divide the 20,000 bit stream into 5000 contiguous 4 bit segments. Count and store the number of occurrences of each of the 16 possible 4 bit values. Denote $g(i)$ as the number of each 4 bit value $i$ where 0 - 15 .

2) Calculate $x$ by

$$
X=\frac{16}{5000} \sum_{i=0}^{15} g(i)^{2}-5000
$$

3) The test is passed if $2.16<x<46.17$.

\subsubsection{The Runs Test}

1) A run represents a maximal sequence of consecutive bits of all ones or all zeros. The incidences of runs of all lengths in the sample stream should be counted and stored.

2) The test is passed if the number of runs is each within the corresponding interval specified below Table 10.

\subsubsection{The Long Run Test}

1) Find the longest run in the 20,000 bits.

2) If the length of the longest run in the bit stream of 20,000 bit (both of one and zero) is smaller than 26 , the test is passed. 
Table 6. Correlation coefficient for plain and cipher image.

\begin{tabular}{ccccccc}
\hline & \multicolumn{2}{c}{ Correlation coefficient of plain image } & \multicolumn{2}{c}{ Correlation coefficient of Cipher image } \\
\hline Images & Horizontal & Vertical & Diagonal & Horizontal & Vertical \\
Baboon & 0.8646 & 0.8293 & 0.8114 & 0.004 & 0.007 & 0.001 \\
Lena & 0.9156 & 0.8808 & 0.8603 & 0.006 & 0.005 & 0.006 \\
Pepper & 0.9376 & 0.9364 & 0.8935 & 0.023 \\
\hline
\end{tabular}

Table 7. Entropy test for different color image.

\begin{tabular}{ccccccccccc}
\hline Image & $R=1$ & $R=2$ & $R=3$ & $R=4$ & $R=8$ & $R=10$ & $R=16 \quad$ Yong Wang et al. [7] \\
\hline \multirow{2}{*}{ Baboon } & 7.9988 & 7.9990 & 7.9991 & 7.9992 & 7.9990 & 7.9990 & 7.9990 \\
Lenna & 7.9981 & 7.9991 & 7.9991 & 7.9992 & 7.9991 & 7.9990 & 7.9990 & 7.9990 & 7.9991 \\
Pepper & 7.9987 & 7.9991 & 7.9991 & 7.9989 & 7.9989 & 7.9992 & 7.9992 & 7.9992 \\
Airplan & 7.9989 & 7.9989 & 7.9991 & 7.9991 & 7.9991 & 7.9991 & 7.9991 & 7.9992 \\
Boat & 7.9986 & 7.9992 & 7.9991 & 7.9990 & 7.9990 & 7.9990 & 7.9991 & 7.9991 \\
\hline
\end{tabular}

Table 8. Encryption time in second for different round.

\begin{tabular}{|c|c|c|c|c|c|c|c|c|}
\hline Image & $R=1$ & $R=2$ & $R=3$ & $R=4$ & $R=8$ & $R=10$ & $R=16$ & $R=32$ \\
\hline \multicolumn{9}{|c|}{ Our Scheme } \\
\hline Baboon & 0.510 & 0.87 & 1.20 & 1.56 & 2.94 & 3.65 & 5.73 & 11.24 \\
\hline Lenna & 0.521 & 0.87 & 1.197 & 1.561 & 2.933 & 3.662 & 5.697 & 11.225 \\
\hline Pepper & 0.521 & 0.87 & 1.197 & 1.561 & 2.933 & 3.662 & 5.697 & 11.225 \\
\hline Airplan & 0.521 & 0.87 & 1.197 & 1.561 & 2.933 & 3.662 & 5.697 & 11.225 \\
\hline Boat & 0.521 & 0.87 & 1.197 & 1.561 & 2.933 & 3.662 & 5.697 & 11.225 \\
\hline
\end{tabular}

Table 9. Decryption time in second for different round.

\begin{tabular}{|c|c|c|c|c|c|c|c|c|}
\hline Image $256 \times 256$ & $R=1$ & $R=2$ & $R=3$ & $R=4$ & $R=8$ & $R=10$ & $R=16$ & $R=32$ \\
\hline \multicolumn{9}{|c|}{ Our Scheme } \\
\hline Baboon & 0.43 & 0.77 & 1.12 & 1.470 & 2.85 & 3.55 & 5.62 & 11.17 \\
\hline Lenna & 0.429 & 0.77 & 1.137 & 1.471 & 2.869 & 3.548 & 5.618 & 11.20 \\
\hline Pepper & 0.430 & 0.78 & 1.139 & 1.472 & 2.869 & 3.549 & 5.619 & 11.22 \\
\hline Airplan & 0.429 & 0.77 & 1.137 & 1.471 & 2.869 & 3.548 & 5.618 & 11.20 \\
\hline Boat & 0.434 & 0.722 & 1.065 & 1.414 & 2.807 & 3.523 & 5.594 & 11.15 \\
\hline
\end{tabular}

We need to change the testing algorithm to suit to image data so we randomly chose 100 streams of 20,000 consecutive bits from the ciphered images of image A. Then we find statistics of the randomly chosen 100 streams for each test and compared them to the acceptance ranges. Table 11 show the numbers of the samples among 100 randomly chosen samples, which passed the Mono-bit, Poker, Long run tests and run tests.

\section{Conclusions}

This paper presents a technique which replaces the tradi- tional preprocessing complex system and utilizes the basic operations like confusion, diffusion which provide same or better encryption using cascading of 3D standard and 3D cat map. We generate diffusion template using 3D standard map and rotate image by using vertically and horizontally red and green plane of the input image. We then shuffle the red, green, and blue plane by using 3D Cat map and standard map. Finally the Image is encrypted by performing XOR operation on the shuffled image and diffusion template. Completion of the design, both theoretical analyses and experimental tests have been carried out, both confirming that the new cipher 
Table 10. FIPS-140 test range.

\begin{tabular}{cccccc}
\hline Length of the run & 1 & 2 & 3 & 4 & 5 \\
\hline Required interval & $2315-2685$ & $1114-1386$ & $527-723$ & $240-384$ & $103-209$ \\
\hline
\end{tabular}

Table 11. FIPS-140 test $P=$ pass, $F=$ fail.

\begin{tabular}{|c|c|c|c|c|c|c|c|c|c|}
\hline Name of image & & $R=1$ & $R=2$ & $R=3$ & $R=4$ & $R=8$ & $R=10$ & $R=16$ & $R=32$ \\
\hline \multirow[b]{2}{*}{ Baboon } & runs & $10 \mathrm{P}, 10 \mathrm{P}$ & $11 \mathrm{P}, 12 \mathrm{P}$ & $13 \mathrm{P}, 15 \mathrm{P}$ & $12 \mathrm{P}, 14 \mathrm{P}$ & $15 \mathrm{P}, 11 \mathrm{P}$ & $12 \mathrm{P}, 12 \mathrm{P}$ & $16 \mathrm{P}, 12 \mathrm{P}$ & $17 \mathrm{P}, 14 \mathrm{P}$ \\
\hline & pocker & $374.7 \mathrm{~F}$ & $9.8944 \mathrm{P}$ & $15.443 \mathrm{P}$ & $12.985 \mathrm{P}$ & $13.644 \mathrm{P}$ & $8.678 \mathrm{P}$ & $12.556 \mathrm{P}$ & $12.556 \mathrm{P}$ \\
\hline \multirow{3}{*}{ Lenna } & runs & $10 \mathrm{P}, 11 \mathrm{P}$ & $13 \mathrm{P}, 15 \mathrm{P}$ & $13 \mathrm{P}, 15 \mathrm{P}$ & $17 \mathrm{P}, 13 \mathrm{P}$ & $12 \mathrm{P}, 12 \mathrm{P}$ & $13 \mathrm{P}, 13 \mathrm{P}$ & $15 \mathrm{P}, 20 \mathrm{P}$ & $12 \mathrm{P}, 12 \mathrm{P}$ \\
\hline & pocker & $317.4 \mathrm{~F}$ & $24.30 \mathrm{P}$ & $14.022 \mathrm{P}$ & 8.9920P & $18.227 \mathrm{P}$ & $21.856 \mathrm{P}$ & $11.558 \mathrm{P}$ & $18.752 \mathrm{P}$ \\
\hline & mono & $9956 \mathrm{P}$ & $10025 \mathrm{P}$ & $10103 \mathrm{P}$ & $9967 \mathrm{P}$ & 9938P & $10054 \mathrm{P}$ & 9900P & $10112 \mathrm{P}$ \\
\hline \multirow{3}{*}{ Pepper } & runs & $12 \mathrm{P}, 16 \mathrm{P}$ & $13 \mathrm{P}, 15 \mathrm{P}$ & $12 \mathrm{P}, 13 \mathrm{P}$ & $12 \mathrm{P}, 14 \mathrm{P}$ & $12 \mathrm{P}, 13 \mathrm{P}$ & $13 \mathrm{P}, 13 \mathrm{P}$ & $13 \mathrm{P}, 11 \mathrm{P}$ & $14 \mathrm{P}, 14 \mathrm{P}$ \\
\hline & pocker & $138.41 \mathrm{~F}$ & $13.196 \mathrm{P}$ & $12.057 \mathrm{P}$ & $13.337 \mathrm{P}$ & $18.227 \mathrm{P}$ & $20.454 \mathrm{P}$ & $10.227 \mathrm{P}$ & 27.929P \\
\hline & mono & $10085 \mathrm{P}$ & 9982P & $10001 \mathrm{P}$ & $9967 \mathrm{P}$ & $10048 \mathrm{P}$ & 9994P & 9913P & $10036 \mathrm{P}$ \\
\hline \multirow{3}{*}{ Airplan } & runs & $12 \mathrm{P}, 10 \mathrm{P}$ & $14 \mathrm{P}, 13 \mathrm{P}$ & $17 \mathrm{P}, 23 \mathrm{P}$ & $12 \mathrm{P}, 14 \mathrm{P}$ & $14 \mathrm{P}, 13 \mathrm{P}$ & $15 \mathrm{P}, 12 \mathrm{P}$ & $12 \mathrm{P}, 13 \mathrm{P}$ & $12 \mathrm{P}, 11 \mathrm{P}$ \\
\hline & pocker & $880.25 \mathrm{~F}$ & $30.016 \mathrm{P}$ & $13.504 \mathrm{P}$ & $12.134 \mathrm{P}$ & $16.761 \mathrm{P}$ & $20.108 \mathrm{P}$ & $12.800 \mathrm{P}$ & $9.568 \mathrm{P}$ \\
\hline & mono & $10030 \mathrm{P}$ & $10075 \mathrm{P}$ & 9975P & 9996P & 9940P & $10071 \mathrm{P}$ & 9946P & $9973 \mathrm{P}$ \\
\hline
\end{tabular}

possesses high security and fast encryption speed. In conclusion, therefore, the new cipher indeed has excellent potential for practical image encryption applications.

\section{References}

[1] G. Chen, Y. Mao and C. K. Chui, "A Symmetric Image Encryption Scheme Based on 3D Chaotic Cat Maps," Chaos Solitons \& Fractals, Vol. 21, No. 3, 2004, pp. 749-761. doi:10.1016/j.chaos.2003.12.022

[2] D. Xiao, X. Liao and P. Wei, "Analysis and Improvement of a Chaos-Based Image Encryption Algorithm," Chaos Solitons \& Fractals, Vol. 40, No. 5, December 2007.

[3] Y. Wanga, K.-W. Wong, X. F. Liao and G. R. Chen, "A New Chaos-Based Fast Image Encryption Algorithm," Applied Soft Computing, Vol. 11, No. 1, 2011, pp. 514-522. doi:10.1016/i.asoc.2009.12.011
[4] Z. Guan, F. Huang and W. Guan, "Chaos-Based Image Encryption Algorithm," Physics Letters A, Vol. 346, No. 1-3, 2005, pp. 153-157. doi:10.1016/j.physleta.2005.08.006

[5] S. Lian, J. Sun and Z. Wang, "A Block Cipher Based on a Suitable Use of the Chaotic Standard Map," Chaos Solitons \& Fractals, Vol. 26, No. 1, 2005, pp. 117-129. doi:10.1016/j.chaos.2004.11.096

[6] K. W. Wong, B. S. Kwok and W. S. Law, "A Fast Image Encryption Scheme Based on Chaotic Standard Map," Physics Letters A, Vol. 372, No. 15, 2008, pp. 2645-2652. doi:10.1016/i.physleta.2007.12.026

[7] Y. Mao, G. Chen and S. Lian, "A Novel Fast Image Encryption Scheme Based on 3D Chaotic Baker Maps," International Journal of Bifurcation and Chaos, Vol. 14, No. 10, 2004, pp. 3613-3624. doi:10.1142/S021812740401151X 